\title{
BIOGÁS: UNA ALTERNATIVA SUSTENTABLE PARA SU IMPLEMENTACIÓN EN LABORATORIOS ESCOLARES RURALES
}

Monzón, Virginia ${ }^{1}$; Mena, Cecilia ${ }^{2}$; Sogari, Noemí ${ }^{3}$

\section{RESUMEN:}

El presente trabajo expone el desarrollo de un proyecto de extensión, sobre el uso de las energías renovables en la escuela. $\mathrm{La}$ interacción de investigadores formados y alumnos de la $\mathrm{FaCeNa}$ de la UNNE, contribuye a concretar uno de los objetivos de la universidad: una educación universitaria inclusiva en el nivel medio. El proyecto se realiza con el objeto de promocionar el uso de las energías renovables y en este caso particular la generación de biogás a partir de la degradación de efluentes de corrales y tambos. Los destinatarios son docentes y alumnos de escuelas del nivel medio asentados en zonas rurales. Se aplica una práctica de laboratorio sencilla para la generación de biogás como estrategia didáctica para lograr un proceso de enseñanza-aprendizaje significativo para los educandos, que vincula conceptos físico- químicos vinculados con degradación del medio ambiente, su conservación y la interpretación de manera crítica de los resultados obtenidos.
Palabras Claves: educación, enseñanza, biogás

\section{INTRODUCCION:}

La práctica experimental es uno de los aspectos más importantes en el proceso de enseñanza y aprendizaje de las ciencias. La misma ofrece la posibilidad a los estudiantes de relacionar teoría y práctica y además desarrollar ciertas habilidades y destrezas.

Las prácticas de laboratorio resultan de gran utilidad para potenciar los objetivos conceptual, relacionados con la apropiación de los conceptos, teorías, relaciones de magnitudes, y procedimental. Estos aspectos relacionados con la metodología científica, ayudan a la promoción de capacidades de pensamiento crítico y creativo, como también al desarrollo de actitudes de objetividad y desconfianza ante aquellos juicios de valor presentados en teoría sin las evidencias necesarias (Hodson, 1993).

1. Alumna. FaCeNa-UNNE-virgmonzon@gmail.com

2. Alumna. FaCeNa-UNNE-cecibmena@gmail.com

3. Directora del Grupo de Investigación GIESMA. Directora del PI Estudio, caracterización y aprovechamiento de las propiedades físico-químicas del biogás generado por biodegradación de residuos efluentes industriales. CyT-UNNEnoemisogari@gmail.com 
Desde el punto de vista del constructivismo, la actividad experimental cumple un papel importante dentro del proceso enseñanza-aprendizaje, si se dirige de manera consciente e intencionada a lograr que las ideas previas de los estudiantes evolucionen a conceptos más elaborados y cercanos a los científicos (Tamayo y Sanmartí, 2007; Tamayo, 2009)

Según lo expresara el físico y premio nobel Feynman Richard "la ciencia es una actividad del hombre para observar hechos, contrarrestarlos, experimentarlos, hacer inferencias, deducir conclusiones, dudar y comprobar los resultados del pasado ya establecidos, enseñando en todo momento el valor de la racionalidad".

Las Ciencias Naturales ofrecen un espectro amplio de fenómenos reales que pueden ser reproducidos bajo condiciones controladas para su análisis y estudio. La explicación de un fenómeno es una tarea que ayuda a la articulación entre teoría y práctica, la construcción de conocimientos, la adquisición de formas de trabajo científico y al desarrollo de actitudes, habilidades y destrezas propias del trabajo experimental. En la actualidad son de especial interés: el aporte del trabajo experimental al desarrollo de habilidades para el trabajo en equipo, el establecimiento de relaciones significativas entre las actividades prácticas propuestas y la vida cotidiana de los estudiantes, y las relaciones entre el campo específico de la actividad práctica (biología, química, física) con otros campos del conocimiento.

La educación ambiental, el uso racional de la energía y el aprovechamiento de las energías renovables son temas presentes en las noticias en virtud de los desastres naturales vistos con frecuencia en el mundo. Promover una mayor conciencia ambiental lleva a la formación de ciudadanos más reflexivos, críticos, activos con espíritu solidario con su entorno social y ambiental. Fomentar no solo el conocimiento, sino también la acción concreta para intentar encontrar soluciones sustentables con el ambiente, es una tarea que no debe escapar al quehacer docente y es por ello que mediante está implementación de esta experiencia en el aula se pretende una educación integral, propiciando sujetos responsables y positivos con la sociedad.

El objetivo principal de la práctica de laboratorio que es "facilitar que los alumnos lleven a cabo sus propias investigaciones, se contribuye a desarrollar su comprensión sobre la naturaleza de la ciencia y su reflexión sobre el propio aprendizaje personal" (Novak, 1990). Es por ello que las prácticas de laboratorio tienen la vinculación de la teoría con la práctica permitiendo que los alumnos comprendan con mayor soltura los conceptos dados en estas asignaturas. El laboratorio es un espacio que les otorga a los alumnos la construcción del conocimiento científico para el desarrollo de habilidades intelectuales y sociales, permite ver la aplicación real de lo estudiado y de relacionar los contenidos adquiridos en instancias anteriores haciendo al aprendizaje significativo. Concediéndole la capacidad de interpretar situaciones diversas, favoreciendo el abordaje de problemáticas desde el enfoque científico, tecnológico, social y ambiental. 
El abordaje del tema de biogás en las escuelas se piensa de una manera simple y didáctica, teniendo en cuenta las dificultades en materiales y de esa manera no solo nos aseguramos el entendimiento del proceso educativo sino también la promoción de la reutilización de materiales de uso cotidiano. Al realizar las prácticas los alumnos podrán armar varios equipos, discutir los resultados obtenidos, viendo las diferencias que pueden aparecer entre mismos y así con la ayuda del docente ver los conceptos aplicados ayudando de esta manera al fortalecimiento del aprendizaje con materiales de fácil acceso y bajo costos.

La visualización de los procesos de degradación de la materia y generación de biogás, facilitan la comprensión de los conceptos inherentes a energías renovables y su aprovechamiento, sustentabilidad ambiental, materia orgánica biodegradable como impulsador de energía, fermentación anaeróbicos y sus fases, desintegración de desechos, tipos de biodigestores.

La aplicación de estas prácticas en la enseñanza busca llamar la atención de los educandos y despertar en ellos un especial interés para la comprensión de la materia, mostrándoles que a partir de materiales que se usan cotidianamente podemos crear situaciones de laboratorio.

Este tipo de experiencias, ayudan y fortalecen el aprendizaje significativo de los alumnos, en distintos establecimientos educativos que no cuentan con los recursos suficientes para acceder a materiales o reactivos exclusivos de laboratorio que por lo general son de alto costo.

\section{METODOLOGIA}

Los talleres de difusión y capacitación en el uso y aprovechamiento de las energías renovables, están destinados para docentes y alumnos de escuelas de niveles medio. Una de las escuelas donde se realizó el taller de construcción de sistemas generadores de biogás a escala laboratorio, usando materiales reciclables fue la EFA “E1 Zanjón” de Quitilipi perteneciente a la localidad del Chaco.

La experiencia consistió en el armado de equipos simples usando botellas, mangueras, selladores. El sistema generador consistió en una botella, que puede ser de plástico o de vidrio, que se carga con el inóculo (estiércol de vaca) llamamos a este primer recipiente zona de retención de la materia orgánica o reactor. Este recipiente se conecta a otra botella mediante el uso de mangueras de suero o de cristal. Esta segunda botella, que actúa como gasómetro, contiene agua, la que se desplaza hacia un tercer recipiente durante el transcurso de la experiencia a medida que se genera el biogás.

Las uniones mangueras-botella se sellan usando silicona (también puede usarse masilla) de modo que quede herméticamente cerrado.

Se deja pasar unos días y las cargas comenzarán a generar gas que irá desplazando el agua contenida en la segunda botella al recipiente colector.

La realización de este trabajo práctico de laboratorio, permitió abordar distintos conceptos físicos y químicos como: volumen, principio de Arquímedes, energías re- 


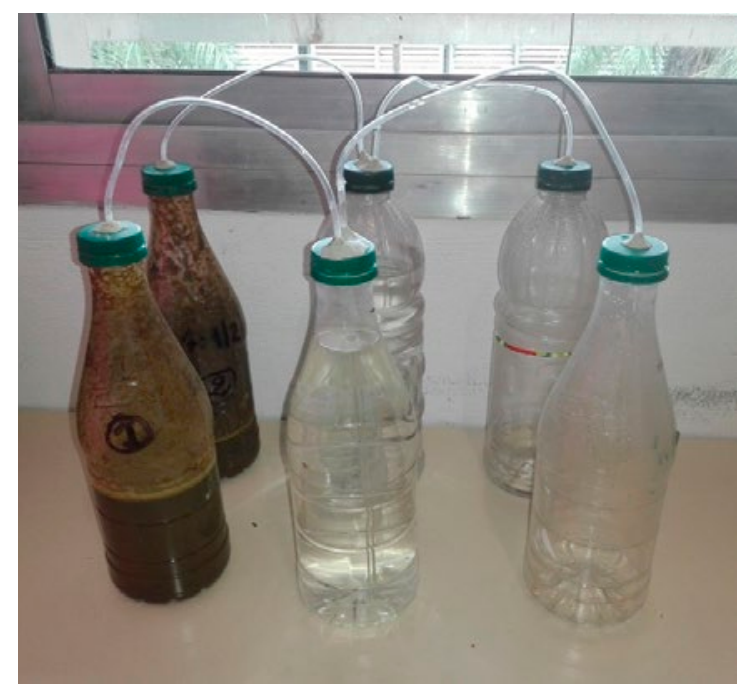

Fig 1: Equipos generadores de biogas usados en la experiencia

novables, diferencia de densidades entre un gas y un líquido, presión, materia orgánica, bacterias, influencia de la temperatura en el proceso de degradación, etc., que le permitirán a los alumnos comprender los diversos temas a partir de una actividad distinta. Como se puede apreciar los conceptos que se pueden desarrollar no corresponden a una sola asignatura, sino que requiere de diferentes áreas, siendo necesario el abordaje interdisciplinario para poder llegar a un fin común que es el aprendizaje de los alumnos y también de los docentes involucrados. Para poder explicar conceptos vinculados con la presión de un gas, por ejemplo, se armó un manómetro.

Como se hace evidente en los equipos que se trabajan son fáciles de armar y económicos pero que mediante su armado los estudiantes podrán trabajar en sus destrezas y habilidades cognitivas para poder realizar la práctica.

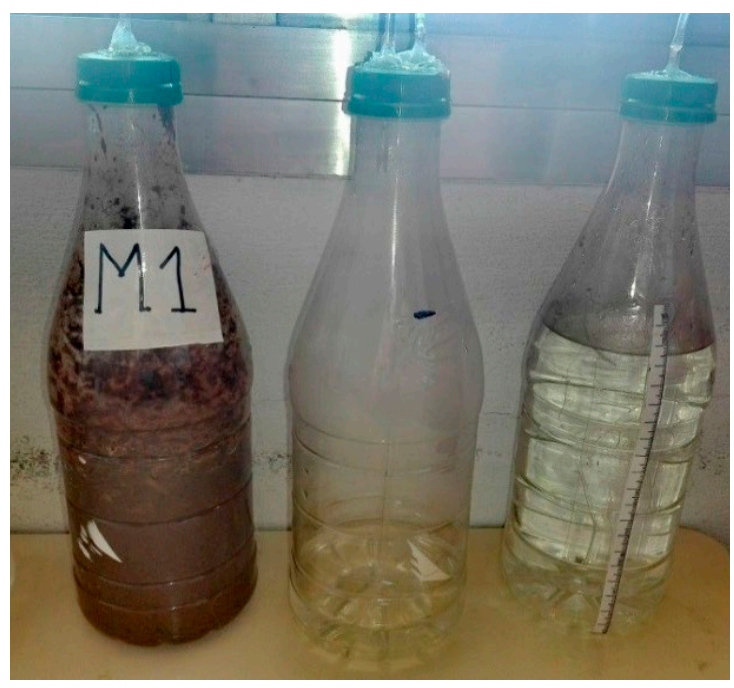

Fig 2: Equipo generador de biogás a escala laboratorio.

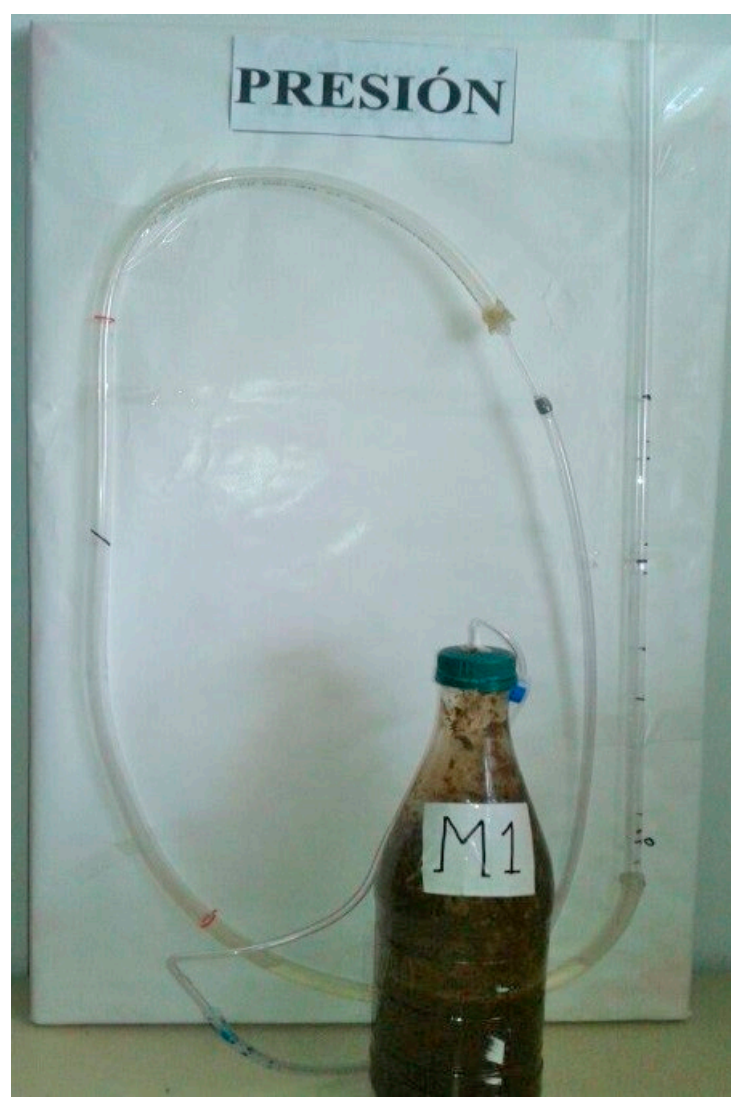

Fig.3 Manómetro realizado con materiales sencillos 


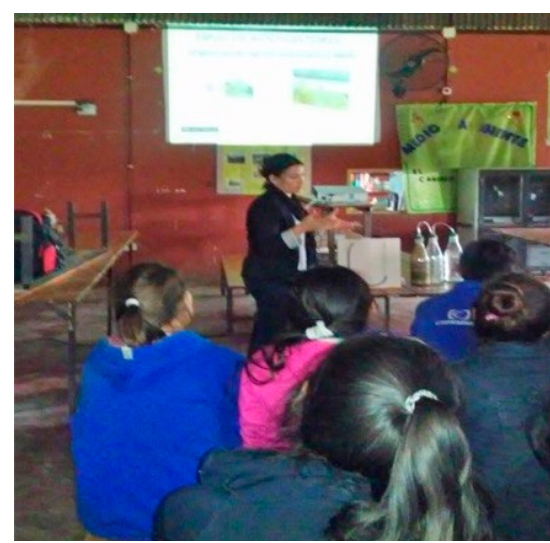

Fig.4 Desarrollo del taller

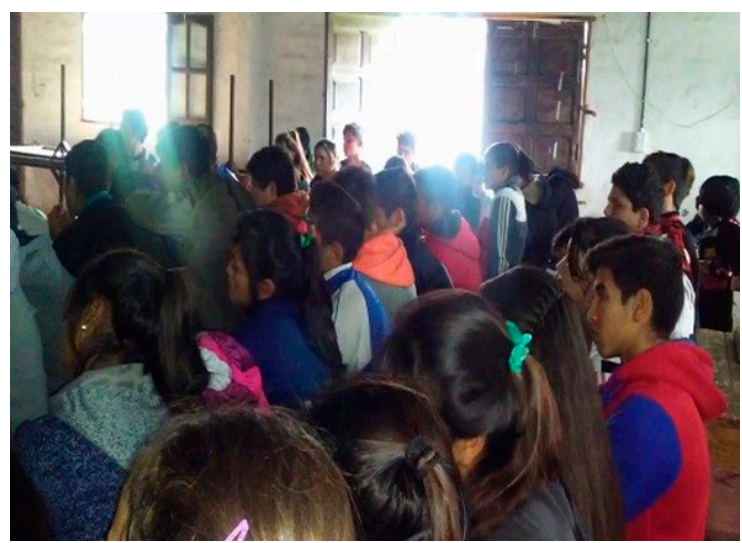

Fig. 5 Alumnos participando del taller

La realización de esta clase de actividades permitió a los educandos adquirir o construir una representación adecuada de lo que está aprendiendo ya que visualiza y manipula con los diferentes conceptos, asegurándonos así una verdadera comprensión y asimilación.

Los alumnos formaron pequeñas comisiones para poder lograr un mejor trabajo colaborativo y ellos mismos armaron los equipos con materiales que llevaron al aula.

\section{CONCEPTOS TRABAJADOS}

La propuesta, además de difundir el uso de las energías renovables para el cuidado del medio ambiente, también permite trabajar interdisciplinarmente con diferentes conceptos integradores relacionados con física, química y matemática. Debido a que mediante de la definición de biogás se pueden trabajar propiedades de los gases en general, separación de fases, efectos de la temperatura, $\mathrm{pH}$ y de esa manera explicar lo que va sucediendo en las diferentes etapas de generación del mismo.
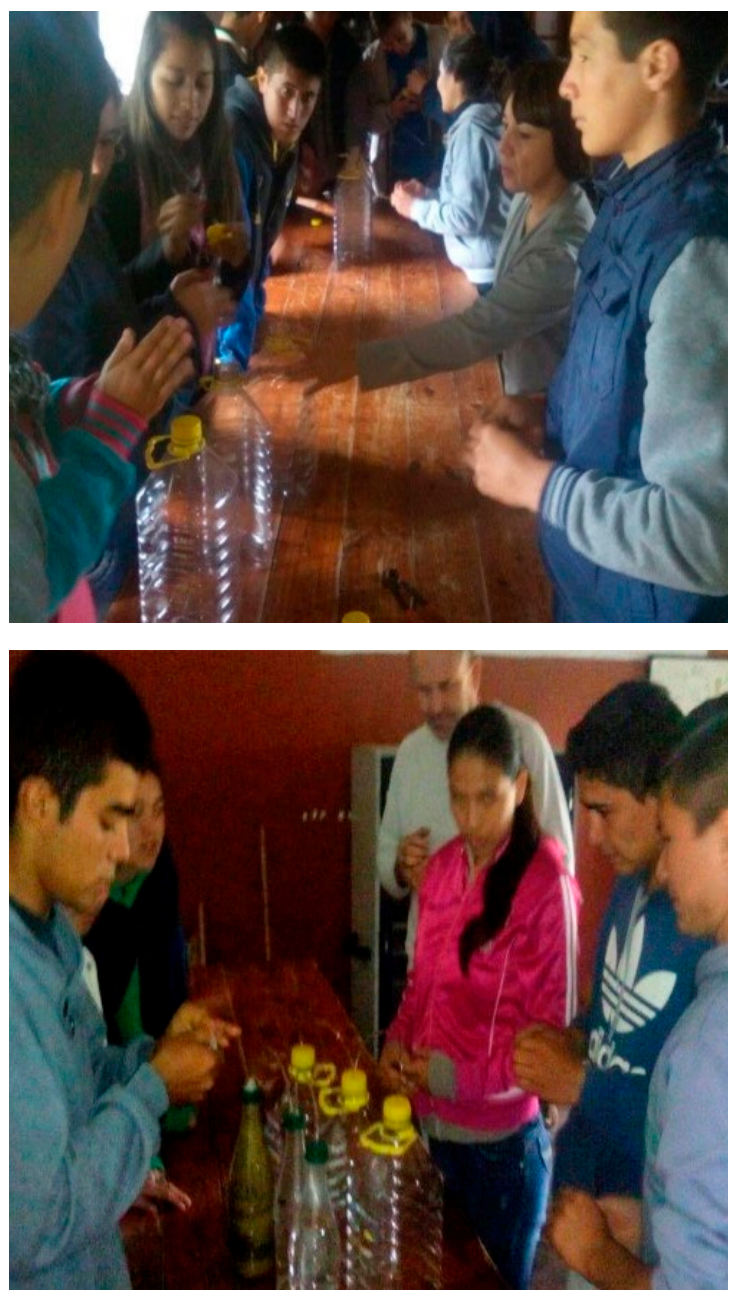

Fig. 6 a y 6b. Alumnos armando sus respectivos biodigestores en pequeñas comisiones 
El biogás, al ser un gas ocupa un lugar en el espacio y es por ello que se puede explicar ese fenómeno a partir del principio de Arquímedes. Esto se evidencia en las botellas descartables y mediante ellos los alumnos pueden reconocer cualitativamente el fenómeno físico.

Se propuso la elaboración de un gráfico de producción de biogás en función del tiempo transcurrido, para ser trabajado con las profesoras de las asignaturas Matemática y Física. Allí se puede vincular definiciones de función, variación del volumen en función del tiempo, velocidad de generación de biogás, etc.

\section{CONCLUSIÓN}

Promover una mayor conciencia ambiental lleva a la formación de ciudadanos más reflexivos, críticos, activos con espíritu solidario con su entorno social y ambiental. La motivación y la curiosidad siempre son los mejores aliados al momento de lograr un aprendizaje significativo y con la implementación en los laboratorios escolares de ésta práctica experimental se pretende mostrar a los alumnos que para el estudio de las energías renovables no es necesario disponer de una gran infraestructura de altos costos, sino que con elementos reciclables y cotidianos, se puede lograr un primer acercamiento al concepto, y así concientizar a cerca del uso racional de la energía, reducir el impacto ambiental y social que generan el uso indiscriminado de recursos agotables.

Promover una mayor conciencia ambiental lleva a la formación de ciudadanos más reflexivos, críticos, activos con espíritu solidario con su entorno social y ambiental.

\section{REFERENCIAS BIBLIOGRAFÍAS}

Davini Cristina. Métodos de Enseñanza. Didáctica general para maestros y profesores. Cap 4 y 5 . Editorial Santillana

Díaz Barriga, M. A. (1985). Didáctica y curriculum; (2ª . Ed.). México, Nuevomar.

Gropelli, Eduardo s. y Giampaoli Orlando a. (2001). El camino de la biodigestión. Ambiente y tecnología socialmente apropiada. 2001.

Hodson, D. (1993). Re-thinking Old Ways: Towards A More Critical Approach To Practical Work In School Science. Journal Studies in Science Education. Vol 22

NAP. (2012) Consejo Federal de Educación. Res CFE 180/12.

Tamayo A., Ó.E. (2009). Didáctica de las ciencias:
La evolución conceptual en la enseñanza y el aprendizaje de las ciencias. Manizales: Editorial Universidad de Caldas.

Tamayo A., Ó.E. y Sanmartí, N. (2007). "HighSchool Students' Conceptual Evolution of the Respiration Concept from the Perspective of Giere's Cognitive Science Model". International Journal of Science Education, No. 2, Vol. 29, pp. 215-248.

Tenreiro, C. y Vieira, R. (2006). "Diseño y validación de actividades de laboratorio para promover el pensamiento crítico de los estudiantes". Revista Eureka sobre Enseñanza y Divulgación de las Ciencias, No. 3, Vol. 3, pp. 452-466, 